\title{
Histomorphometric and Immunohistochemical Characteristics of Oral Melanotic Macule- Overlap with Oral Mucosal Lentigo
}

\author{
Irit Allon*1,3,4, Anna Shnaiderman- Shapiro ${ }^{1}$, Hana Vered ${ }^{1}$, Ilana Kaplan ${ }^{1,2}$ and Abraham Hirshberg ${ }^{1}$ \\ ${ }^{1}$ Department of Oral Pathology \& Oral Medicine, Goldschleger School of Dental Medicine, Tel-Aviv University, Israel \\ ${ }^{2}$ Institute of Pathology, Rabin Medical Center, Petach-Tiqva, Israel \\ ${ }^{3}$ Barzilai Medical center, Ashkelon and Ben-Gurion University, Beer Sheba, Israel \\ ${ }^{4}$ Faculty of Health Sciences, Ben-Gurion University of the Negev, Beer-Sheba, Israel
}

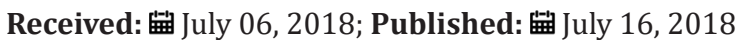

*Corresponding author: Irit Allon, Department of Oral Pathology and Oral Medicine, School of Dental Medicine, Tel Aviv University, Tel Aviv 69978, Israel

Abstract

Aim: Oral melanotic macule is a well-circumscribed pigmented lesion that commonly occurs in the oral mucosa. Histologically, it is characterized by increased production of melanin by basal melanocytes which present normal number and normal morphologic features. Lesions may also exhibit melanin - laden macrophages in the adjacent lamina propria. Because of the benign histological appearance, most authorities ignore its pathogenesis. The purpose of the present study was to immunomorphologically characterize oral melanotic macules.

Materials and Methods: Cases of oral melanotic macule were included and compared with normal appearing margins of the lesion and as reactive hyperplastic lesions as controls. The morphological assessment was carried out by measurement of rete ridge lengths and by immunomorphometric analysis of the epithelial expressions of S100 and HMB45.

Results: The maximal epithelial width of oral melanotic macules was significantly higher than that of the controls. In addition, the frequency of expression of S100 and HMB 45 in OMM differed significantly from the controls; S100 was increased, and HMB45 was decreased.

Conclusions: We suggest that lesions in the spectrum of oral melanotic macule may represent the oral counterpart of skin lentigo, which is characterized by elongation of rete pegs. Higher number of S100 positive cells and low expression of HMB45 suggest an increased number of melanocytes with which lack melanogenic activity.

Keywords: Oral Melanotic Macule; S100; HMB45; Rete Ridge; Lentigo; Neural Crest; Epithelium; Subepithelial Connective Tissue; Wilcoxon Paired; Melanin Incontinence.

\section{Introduction}

Oral melanotic macule (OMM) is usually well circumscribed, predominantly brown and black macule, located mainly on the vermilion border of the lips, followed by the palate, gingiva, and buccal mucosa [1]. Its primary significance is in the differential diagnosis with other solitary pigmented lesions, mainly malignant melanoma. OMMs comprise $0.4-0.7 \%$ of biopsies from oral cavity and perioral tissues [1-3]. The majority (86.1\%) of solitary melanocytic lesions are OMM [1]. Labial melanotic macules develop most frequently in young adults, whereas intraoral lesions are usually noted in patients over the age of 40 . All melanotic macules occur more often in women than in men [2]. The etiology in most cases is unknown, but some labial lesions can be explained by sun exposure and are therefore analogous to "ephelides", as both are morphologically identical. Not all OMMs, however, can be explained by sun exposure, and the lesions do not tend to darken with sun exposure [4]. Some melanotic macules may represent post inflammatory pigmentations of internal or external origin such as Lichen planus or following radiation therapy [5-6] respectively. OMMs may also occur in HIV infected patients, sometimes as a result of medications, trauma, and infection [7].

Histopathologically, the OMMs are characterized by hypermelanosis in the basal cell layer and or lamina propria [3]. Several authors consider the OMM to be the counterpart of cutaneous lentigo, however, in contrast to simple lentigines, OMMs are not reported to exhibit elongation of the rete ridges. Controversies exist concerning the number and function of melanocytes in OMM, while some reports [8] found an increase in the number of dendritic melanocytes; others have reported a lack of melanocytic activity [4,9]. S100 proteins are normally present in cells derived from the neural crest and as such can be used to identify melanocytes in the epithelium. HMB45 antibodies are specifically targeted at melanosomes and are currently used mainly 
to identify melanoma [10]. The purpose of the present study was to characterize OMM, morphologically and immunohistochemically, with special reference to the length of the rete ridges and the expression of melanocytic markers S100 and HMB 45.

\section{Materials and Methods}

The archive of the Department of Oral Pathology and Medicine School of Dental Medicine, Tel-Aviv University was searched for cases diagnosed as OMM. The study has been reviewed and approved by the Tel-Aviv University Ethics Committee. The original slides were reviewed and the diagnoses were confirmed by two of the researches, both, certified oral pathologists (IA, AH). The control group consisted of cases of reactive fibrous hyperplasia. Also, the healthy margins of the OMM lesions were also considered as controls. From each paraffin-embedded block, one slide was stained with H\&E, and two slides were used for the immunohistochemical studies. The histopathological criterion used to diagnose OMM was the accumulation of melanin pigment in the basal and parabasal layers of an otherwise normal epithelium. Melanin may have been free (melanin incontinence) or within melanophages in the upper lamina propria (subepithelial connective tissue).

\section{Morphological and Immunohistochemical Procedures}

For each case, the maximal width of the epithelium was measured by Cell sense, Olympus software from the superficial keratin layer to the bottom of the rete ridge (w-lesion). Measurements were carried out in the center and the margin of the lesions (w-margin) (Figure 1). In addition, the lesions were immunohistochemically stained to assess the expression of S100 and HMB45 in the epithelium. S100 corresponds to the number of melanocytes, while HMB45 may correlate with stimulated melanocytic proliferation and production of melanosomes. $5 \mu \mathrm{m}$ thick sections were cut from formalinfixed, paraffin-embedded tissue blocks on positively charged glass (Superfrost Plus, Fisher Scientific, USA) and stained with primary antibodies as detailed in Table 1 . This was followed by exposure to secondary antibody (Super Picture Poly HRP Conjugate, Invitrogen, Camarillo, CA, USA) for 30 minutes at $250 \mathrm{C}$, then by staining with AEC (RED) substrate kit (Invitrogen, Carlsbad, CA, USA), and finally by staining with Mayer's hematoxylin (Research Chemicals, Colchester, UK).

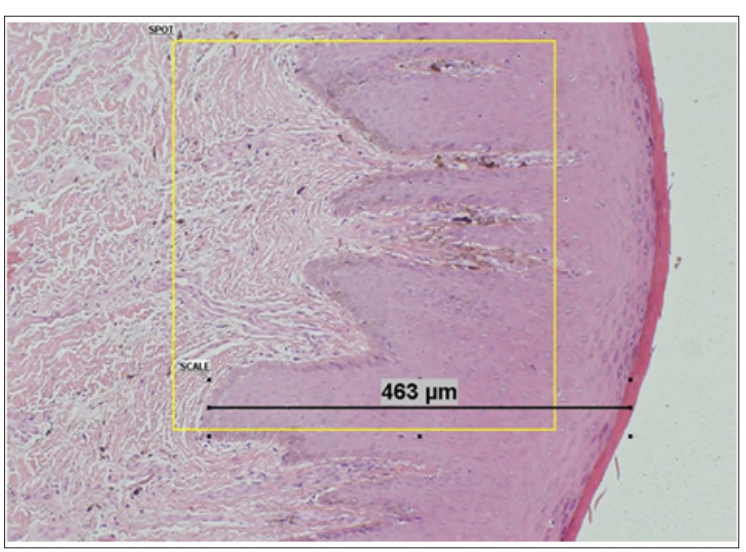

Figure 1: Method of measuring the maximal epithelial width by the DP controller software. For each case, the epithelium was measured by choosing the maximal width of the epithelium rete ridge from top to bottom.
Table 1: Details of the immuno histochemical procedures.

\begin{tabular}{|c|c|l|c|c|}
\hline Protein & Antibody & Manufacturer & Pretreatment & $\begin{array}{c}\text { Positive } \\
\text { control }\end{array}$ \\
\hline S100 & $\begin{array}{c}\text { Monoclonal } \\
1: 2000\end{array}$ & Dako, CA, USA & $\begin{array}{c}\text { Proteinase } \\
\text { K 0.1\% 10 } \\
\text { minutes 37 C }\end{array}$ & Schwannoma \\
\hline HMB45 & $\begin{array}{c}\text { Monoclonal } \\
1: 50\end{array}$ & Dako, CA, USA & $\begin{array}{c}\text { EDTA PH9 } \\
\text { overnight }\end{array}$ & $\begin{array}{c}\text { Malignant } \\
\text { melanoma }\end{array}$ \\
\hline
\end{tabular}

S100 was considered positive when nuclear staining was apparent and HMB45 was considered positive when cytoplasmic staining was obvious, not confused with the granular pigment positivity expected to be present in OMM. The expression of S100 and HMB45 was calculated as the percentage of positive cells out of the total number of the basal cells, counted in 10 consecutive HPFs $(x 400) \pm$ SD. Two types of controls were used. The first, normallooking epithelium at the margins of benign oral lesions (mainly focal fibrous hyperplasia).All cases matched for age, gender and site. In addition the epithelium at the margins of the OMMs was used as control for the width of the rete pegs, only when the margins were not been affected by the lesion.

\section{Statistical Analysis}

The data for each case consisted of several (2-10) frames. In each frame the total number of basal cells and number of S100 and HMB45 cells was calculated. The number of frames was defined by size of the extracted tissue. The parameter of interest was the proportion of S100 and HMB45 positive cells out of total population of basal cells. We took the ratio of total number of S100 and of HMB45 positive cells to the total number of basal cells as the best estimator of the proportion of interest. The distribution data are presented by number of non-missing observations, mean, minimum, maximum, standard deviation and median SD. Logistic regression was used for comparisons of frequency of S100 and HMB45 positive cells among all basal cells between groups. For proportion of HMB45 cells sensitivity analysis was performed using exact Poisson regression with robust estimate of variance and log (total number of basal cells) as offset since the number of HMB45 cells was very low. The width and margins were compared using paired t-test. As a sensitivity analysis, the Wilcoxon paired rank test was added. All p-values are two-sided. P-values below 0.05 were considered as significant. All calculations were done using STATA SE 12 software.

\section{Results}

Overall, 39 patients with OMM were included in the study. The age range was 9-97 years (mean 38.5 years) with 1.2:1 male to female ratio. The most prevalent site was the lip vermilion (LMM) followed by palate, buccal mucosa and gingiva. The mean size of the lesion was $4.12 \mathrm{~mm}$. The duration of the lesion ranged from one month to four years. In regard to epithelial width, w-lesion was significantly greater than that of margin width $(332.7 \pm 19.3$ Vs $262.4 \pm 16.1)$. The difference $(70.2 \pm 17.0)$ was highly significant $(\mathrm{p}<0.001$, paired t-test). The two parameters were positively correlated (Pearson correlation 0.5487, $\mathrm{p}<0.001$ ). The relationship between two parameters is shown in Figure 2. 


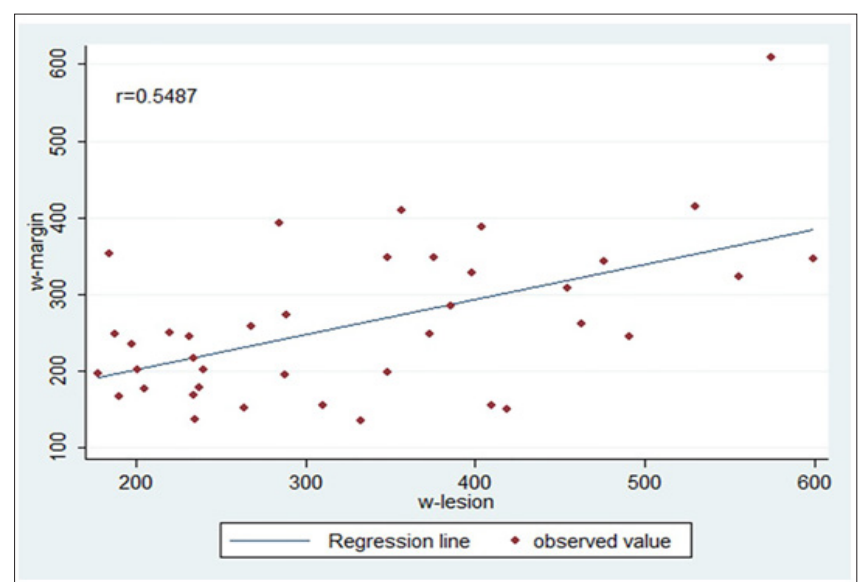

Figure 2: Relationship between w-lesion and w-margin. The observed values of $\mathrm{w}$ - lesion and $\mathrm{w}$ - margin are presented in the scatter. The two measures are significantly positively correlated (Pearson correlation $0.5487 \mathrm{p}<0.001$ ).

\section{Immunohistochemical Analysis}

The immunomorphological features of OMMs versus controls are presented in Table 2. The results show that the expressions of S100 and HMB 45 in OMM were significantly different than in controls, higher for S100 and lower for HMB45, respectively. Mean expression of S100 was $8.67 \% \pm 5.37$ in the OMM group (Figure 3) versus $5.15 \% \pm 4.33$ in the fibrous hyperplasia control $(\mathrm{P}<0.001)$. The expression of HMB45 was generally low and even lower in OMM than controls; the mean expression was $0.18 \% \pm 0.31$ in the OMM (Figure 4) versus $0.49 \% \pm 1.1$ in the controls $(\mathrm{P}=0.003)$.

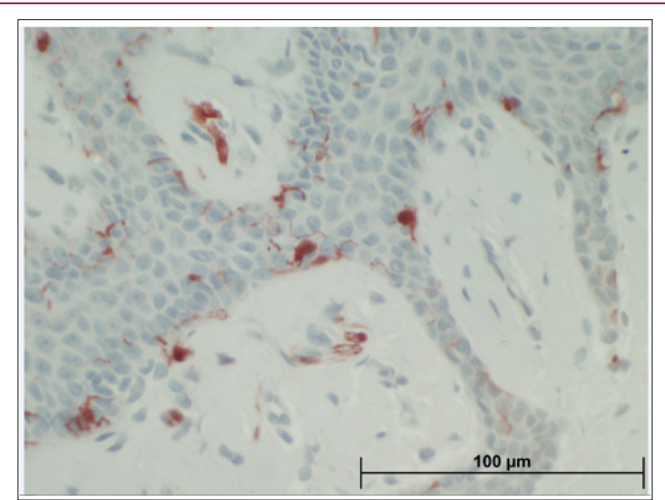

Figure 3: Increased S100 reactivity in oral melanotic macule. Melanocytes in the epithelium as well as melanophages in the lamina propria are positively stained.

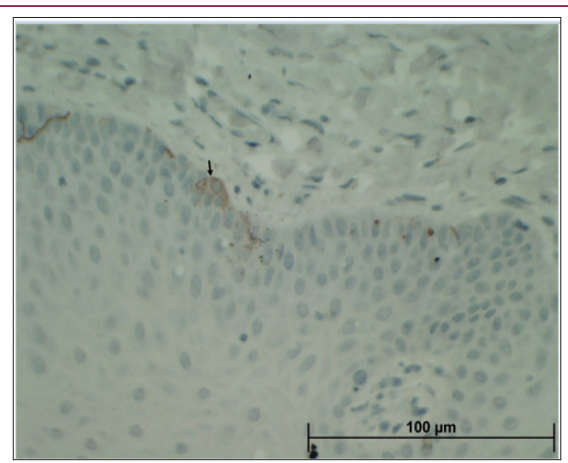

Figure 4: Scattered HMB45 positivity in oral melanotic macule appears different than the granular positivity of melanin pigment, obviously present in oral melanotic macule.

Table 2: Distributions of measured parameters in cases and controls.

\begin{tabular}{|c|c|c|c|c|c|c|}
\hline Group & Statistic & N Basal & N HMB45 & N S100 & Proportion HMB45 & Proportion S100 \\
\hline Control & Mean \pm SD & $525.33 \pm 243.56$ & $1.75 \pm 2.99$ & $31.17 \pm 34.33$ & $.005 \pm .0111$ & $.052 \pm .0432$ \\
\hline OMM & Mean \pm SD & $448.03 \pm 247.88$ & $.56 \pm 1.74$ & $42.077 \pm 36.025$ & $.001 \pm .0031$ & $.087 \pm .0542$ \\
\hline Total & Mean \pm SD & $466.22 \pm 246.69$ & $.84 \pm 2.13$ & $39.51 \pm 35.6$ & $.002 \pm .006$ & $.078 \pm .053$ \\
\hline
\end{tabular}

${ }^{1} \mathrm{P}=0.001$

${ }^{2} \mathrm{P}<0.001$

\section{Discussion}

OMMs are pigmented, usually unifocal lesions that are histopathologically characterized by increased production of melanin by basal melanocytes [11]. Melanin may also be observed in melanophages in the upper portion of the lamina propria $[8,12]$. Some authors, however, define OMM as a lesion that may display an increased number of melanocytes along the junctional zone, especially when they appear on the lips [8]. Buery and co-workers investigated the immunophenotype of OMMs along with other benign and malignant melanocytic lesions. S100 was detected in most benign lesions while in OMM, only sporadic positive HMB45 cells were found S-100 corresponds to the number of melanocytes, while HMB-45 may correlate with stimulated melanocytic proliferation and production of melanosomes) In the present study, the expression of S100 was higher than in controls, with only scattered HMB45 positive cells detected, which were even lower in OMM than in the control group.
Lentigo simplex (LS) is a benign pigmented melanocytic lesion which is one of several forms of benign melanocytic hyperplasia of unknown cause. Unlike ephelis, LS is typically found on areas not exposed to sunlight, is somewhat darker in color, does not darken with sun exposure, and is believed to represent an increase in both local melanin production and the number of melanocytes. Oral lesions of LS have been reported in the literature, but their occurrence is regarded as uncommon and when they occur, may be accepted as a form of OMM. In the present study, we have shown an increased epithelial width in cases of OMM, which may support the assumption that at least some OMM cases can be viewed as oral counterpart of cutaneous lentigo. The morphology and density of melanocytes in the OMM has not been well studied. Melanocytes on $\mathrm{H} \& \mathrm{E}$ sections are difficult to distinguish from intramucosal lymphocytes, which are frequently present in the oral mucosa. The purpose of the present study was, therefore, to characterize OMM, morphologically and immunohistochemically. Morphologically, 
we aimed at evaluating the extent of epithelial hyperplasia by measuring the maximal length of the epithelial rete ridges in the lesion versus controls. A significant difference between the maximal widths of the epithelium in OMM versus healthy appearing margins was found. This difference in the maximal width could suggest that at least some OMMs may represent a form of cellular hyperplasia.

To appreciate if the hyperplasia is due to the melanocytic component, the expression of S100 and HMB45 was evaluated as percentage of the entire basal cell population. The S100 antigen is the most common marker used for melanocyte detection, and its higher expression in OMMs has been previously documented in the literature, and supported in the present study. The higher percentage of S100 positive cells found in the present study could be attributed to melanocytic hyperplasia. HMB45 reactivity was documented in OMMs of HIV patients [7]. This monoclonal antibody directed against a melanosomal glycoprotein is thought to be expressed by melanocytes that lack all melanogenic activity. The present results found a lower HMB45 expression in OMMs compared to controls. This finding may reflect a higher melanogenic activity compared to controls. Histopathologically, OMMs were traditionally characterized by absence of rete ridge elongation and lack of prominent melanocytic activity, and HMB45 immunoreactivity was typically lacking $[4,9,11,13]$.

In contrast, Sexton et al. found that OMMs do present an increase in the number of melanocytes [8] as reflected by the results of the present study. HMB45 immunostain in the present study supports the finding of scattered melanocytes in normal oral mucosa and even lower expression in OMM. The same results were found in other sun-protected sites, for example in the anorectal mucosa in which the HMB45 immunostain in areas of benign melanocytic hyperplasia was found to be very low compare with that found in control squamous mucosa [14]. In light of this, we suggest that lesions in the spectrum of OMM could represent an overlap with lesions characterized by melanocytic hyperplasia/ rete elongation such as lentigo. The increase in the epithelial length (elongation of rete ridges), as well as the increase in S100 positive cells (representing melanocytic hyperplasia), with a minute decrease in HMB45 [15-24] in cases of OMM, may indicate that at least in some cases there are similar histological characteristics with cutaneous LS. OMM probably represent a form of oral lentigo rather than a separate entity.

\section{References}

1. Buchner A, Hansen LS (1979) Melanotic macule of the oral mucosa: A clinic pathological study of 105 cases. Oral Surg Oral Med Oral Pathol 48(3): 244-249.

2. Buchner A, Merrel PW, Carpenter WM (2004) Relative frequency of solitary melanocytic lesions of the oral mucosa. J Oral Pathol Med 33(9): 550-557.

3. Kaugars GE, Heise AP, Riley WT, Abbey LM, Svirsky JA (1993) Oral melanotic macules: A review of 353 cases. Oral Surg Oral Med Oral Pathol Oral Radiol Endod 76(1): 59-61.

4. Weathers DR, Corio RL, Crawford BE, Giansanti JS, Page LR (1976) The labial melanotic macule. Oral Surg Oral Med Oral Pathol 42(2): 196-205.
5. Barrett AW, Porter SR, Scully C, JW Eveson, MJ Griffiths (1994) Oral melanotic macules that develop after radiation therapy. Oral Surg Oral Med Oral Pathol 77(4): 431-434.

6. Barrett AW, Scully C (1994) Human oral mucosal melanocytes: a review. J Oral Pathol Med 23(3): 97-103.

7. Ficarra G, Di Lollo, G Asirelli, Rubino I (1995) Melanocytic activation in HIV disease: HMB-45 positivity in oral melanotic macules. Oral Surg Oral Med Oral Pathol Oral Radiol Endod 1995 80(4): 457.

8. Sexton FM, Maize JC (1987) Melanotic macules and melanoacanthomas of the lip: A comparative study with census of the basal melanocyte population. Am J Dermatopathol 9(5): 438-444.

9. Page LR, Corio RL, Crawford BE, JS Giansanti, DR Weathers (1977) The oral melanotic macule. Oral Surg Oral Med Oral Pathol 44(2): 219-226.

10. Taatjes DJ, Arendash-Durand B, von Turkovich M, Trainer TD (1993) HMB-45 antibody demonstrates melanosome specificity by immunoelectron microscopy. Arch Pathol Lab Med 117(3): 264-268.

11. Eisen D (2000) Disorders of pigmentation in the oral cavity. Clin Dermatol 18(5): 579-587.

12. Ho KK, Dervan P, O’Loughlin S, et al. (1993) Labial melanotic macule: A clinical, histopathologic, and ultrastructural study. J Am Acad Dermatol 28(1): 33-39.

13. Kauzman A, Pavone M, Blanas N, Bradley G (2004) Pigmented lesions of the oral cavity: review, differential diagnosis, and case presentation. J Can Dent Assoc 70(10): 682-683.

14. Tse JY, Chan MP, Zukerberg LR, Nazarian RM (2016) Assessment of Melanocyte Density in Anorectal Mucosa for the Evaluation of Surgical Margins in Primary Anorectal Melanoma. Am J Clin Pathol 145(5): 626634.

15. Çiçek Y, Ertas, U“ (2003) The normal and pathological pigmentation of oral mucous membrane: a review. J Contemp Dent Pract 4(3): 76-86.

16. Fitzpatrick TB, Breatnach AS (1963) Das epidermale melanineinheitsystem. Dermatol Wschr 147: 481-489.

17. Grichnik JM, Rodhes A, Sober AJ (2003) Benign hyperplasias and neoplasias of melanocytes. In: Freedberg A, Eisen K, Wolff K, Austen L Goldsmith S, Katz (Eds.). Fitzpatrick's dermatology in general medicine ( $6^{\text {th }}$ Edn.)., McGraw Hill, New York, USA, p. 882.

18. Hafner C, Stoehr R, Van Oers JM, Zwarthoff EC, Hofstaedter F, et al. (2009) The absence of BRAF, FGFR3, and PIK3CA mutations differentiates lentigo simplex from melanocytic nevus and solar lentigo. J Invest Dermatol 129(11): 2730-2735.

19. Hicks MJ, Flaitz CM (2000) Oral mucosal melanoma: epidemiology and pathobiology. Oral Oncol 36(2): 152-169.

20. Langford A, Pohle HD, Gelderblom X, Reichart PA (1989) Oral hyperpigmentation in HIV-infected patients. Oral Surg Oral Med Oral Pathol 67(3): 301-307.

21. Lenane P, Powell FC (2000) Oral pigmentation. J Eur Acad Dermatol Venereol 14(6): 448-465.

22. Meyerson MA, Cohen PR, Hymes SR (1995) Lingual hyperpigmentation associated with minocycline therapy. Oral Surg Oral Med Oral Pathol Oral Radiol Endod 79(2): 180-184.

23. Mirowski GW, Waibel JS (2002) Pigmented lesions of the oral cavity. Dermatol Ther 15: 218-228.

24. Yanagy K, Haneji N, Ishimaru N, Hamano H, Hayashi Y (1996) Immuno pathological analysis of mucosal melanocyte distribution in the human lower lip of the elderly. Pathobiol 64(3): 156-160. 


\section{ISSN: 2574-1241}

DOI: 10.26717/BJSTR.2018.06.001414

Irit Allon. Biomed J Sci \& Tech Res

(c) (i) This work is licensed under Creative

Submission Link: https://biomedres.us/submit-manuscript.php

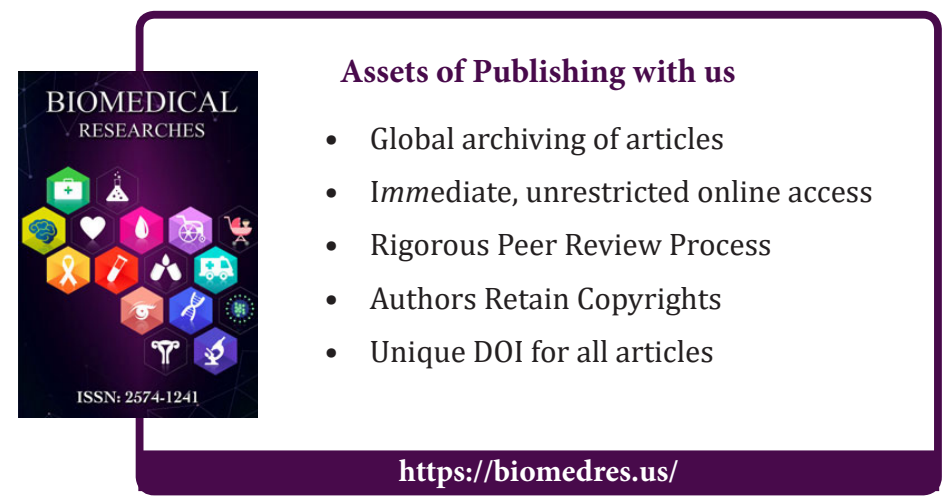

